\title{
Search for New Physics at HERA
}

\author{
Andrea Parenti ${ }^{1}$ \\ Notkestraße 85, 22607 Hamburg, Germany
}

\begin{abstract}
The HERA $e^{ \pm} p$ collider operated between 1992 and 2007. The two colliding beam, general purpose experiments, H1 and ZEUS, collected together and integrated luminosity of $\sim 1 \mathrm{fb}^{-1}$. In the present paper it is presented a summary of the the most recent results on the search for new physics the above mentioned experiments. Most of the results have been obtained by making use of the full available statistics.
\end{abstract}

Keywords: ep collisions, new physics, searches

PACS: $12.60 .-\mathrm{i}, 13.60 .-\mathrm{r}, 14.65 . \mathrm{Ha}, 14.80 .-\mathrm{j}$

\section{INTRODUCTION}

The HERA $e^{ \pm} p$ collider was operated between 1992 and 2007. Electron or positron beams with an energy of $27.6 \mathrm{GeV}$ were collided with proton beams with an energy up to $920 \mathrm{GeV}$, resulting in a center-of-mass energy $\sqrt{s}$ up to $319 \mathrm{GeV}$. The two collidingbeam general purpose experiments, $\mathrm{H} 1$ [1, 2] and ZEUS [3], collected an integrated luminosity of about $0.5 \mathrm{fb}^{-1}$ each.

\section{SEARCH FOR NEW PHYSICS AT HERA}

At HERA the Deep Inelastic $e^{ \pm} p$ Scattering (DIS) processes proceed via the exchange of an electro-weak boson, that can be either neutral $\left(\gamma, Z^{0}\right)$ or charged $\left(W^{ \pm}\right)$. We will talk about Neutral Current (NC) processes in the first case, of Charged Current (CC) processes in the latter. The virtuality of the boson, $Q^{2}$, sets the scale at which the proton is probed. New Physics, if exists, is expected to appear at high energies (small distances). At HERA we reached $Q^{2}$ values bigger $10^{4} \mathrm{GeV}^{2}$, which corresponds to a resolving power of the boson of $\sim 10^{-18} \mathrm{~m}$.

The first effect we can look for at HERA are deviations in the Neutral Current DIS cross section. If the quark radius is not zero, we would expect deviation from the Standard Model (SM) at high- $Q^{2}$, described by the additional quark form factor. Both $\mathrm{H} 1$ and ZEUS have compared their cross section measurements to the SM predictions [4, 5] and found no deviations. Therefore upper limits for the quark radius have been derived at 95\% Confidence Level (CL): $R_{q}<0.74 \times 10^{-18} \mathrm{~m}(\mathrm{H} 1), R_{q}<0.62 \times 10^{-18} \mathrm{~m}$ (ZEUS).

Any new interaction appearing at a much higher energy than the center-of-mass can be described in terms of a contact interaction, expected to be visible as deviations from the SM cross section. Since no deviations where observed by ZEUS [4] and H1 [6],

\footnotetext{
${ }^{1}$ On behalf of the H1 and ZEUS Collaborations.
} 
limits where set at $95 \%$ CL on the effective scale of the new interaction. Depending on the chiral structure assumed, limits where set between 2.0 and $8.0 \mathrm{TeV}$ in the ZEUS analysis, and between 1.6 and $5.5 \mathrm{TeV}$ in the $\mathrm{H} 1$ analysis.

Another effect of fermion compositeness would be the existence of excited states of fermions. Such particles would decay to an ordinary fermion by emitting a gauge boson. The $\mathrm{H} 1$ collaboration investigated all the possible decay channels $[7,8,9]$ and found no deviation from the SM prediction. Therefore upper limits were set on the coupling constants of excited to ordinary fermions, at 95\% CL; from these limits lower limits on the excited fermion mass were derived. The mass limits ranged from $\sim 200 \mathrm{GeV}$ to $\sim 270 \mathrm{GeV}$.

Many extensions to the SM predict the existence of leptoquarks (LQs), particles carrying both baryon and lepton numbers; they would have fractional electric charge and SU(3) color. The LQs are explored in the Buchmüller, Rückl and Wyler model [10]. At HERA they can be produced resonantly ( $s$-channel exchange), or they can be exchanged between the incoming lepton and a quark coming from the proton ( $t$-channel exchange). They are expected therefore to decay in electron-jet or neutrino-jet. H1 searched for LQs in NC and CC DIS data [11], and observed no excess over the SM prediction. Therefore upper limits were set on the coupling of LQs to ordinary leptons and quarks. These limits resulted to be competitive with the ones from LEP and TEVATRON experiments.

Events containing high energy leptons (electrons or muons) and missing transverse momentum $\left(P_{T}\right)$ produced in high energy particle collisions are interesting as they may be a signature of physics beyond the SM. In the SM, the production of single $W$ bosons with subsequent leptonic decay gives rise to this topology. The process has been studied by both the H1 [12] and ZEUS [13] collaborations. The H1 collaborations observed some excess in the $e^{+} p$ collisions, in the region of high hadronic transverse momentum, $P_{T}^{X}>25 \mathrm{GeV} .17$ events were observed in data, whereas $8.0 \pm 1.3$ are expected from the $\mathrm{SM}$; the excess corresponds to 2.4 standard deviations. No excess was observed by H1 in the $e^{-} p$ collisions, neither by ZEUS.

Isolated leptons (electrons or muons) with missing $P_{T}$ are also the signature for single top production. In the SM single top can be produced in CC interactions, but the cross section is very small, $O(1 \mathrm{fb})$. Flavor Changing NC processes, if existing, would contribute to the cross section. Both the collaborations have compared the data to the SM predictions $[14,15]$ and found no significant deviations. Therefore limits were set on the anomalous coupling $\kappa_{t u \gamma}$ at $95 \% \mathrm{CL}$; the more stringent result is from ZEUS: $\kappa_{t u \gamma}<0.13$, that is competitive to the limits from the TEVATRON.

At HERA also the production of two or more high- $P_{T}$ leptons (electrons or muons) have been investigated. The two collaborations have combined their data [16], and compared them to the prediction from the SM. The bigger contribution in the SM comes from the so called Bethe-Heitler process, where lepton pairs are produced via $\gamma \gamma$ interactions. The distribution of the scalar sum of all lepton's momenta $\left(\sum P_{T}\right)$ is plotted in Fig. 1, separately for $e^{+} p$ and $e^{-} p$ collisions. In $e^{+} p$ collisions, 7 events with $\sum P_{T}>100 \mathrm{GeV}$ are observed, whereas $1.94 \pm 0.17$ events are predicted by the SM Monte Carlo; this excess corresponds to 2.6 standard deviations. No events with $\sum P_{T}>100 \mathrm{GeV}$ are observed in $e^{-} p$ collisions, whereas $1.19 \pm 0.12$ are expected.

The $\mathrm{H} 1$ collaboration performed a general search for new physics [17]. Events with at least one high- $P_{T}$ electron, photon, muon, neutrino or jet are selected, and divided 

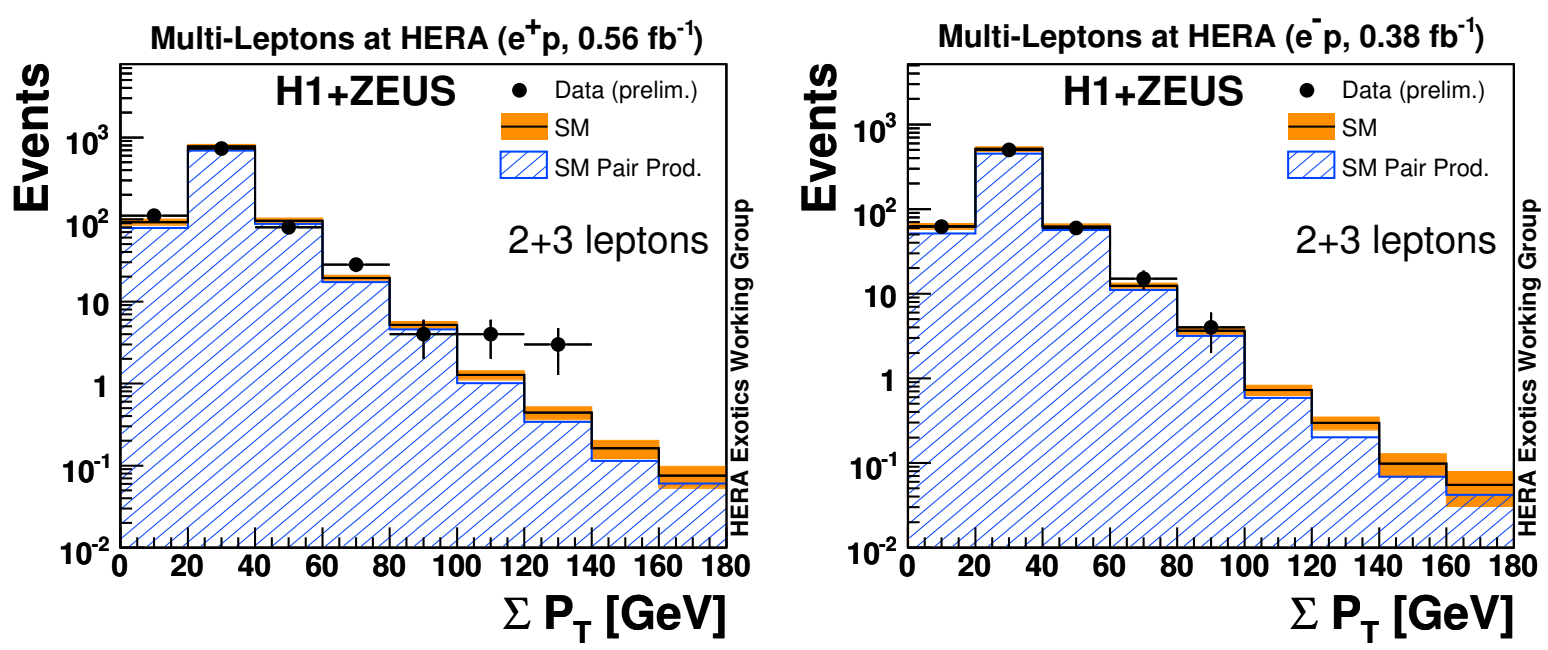

FIGURE 1. The distribution of the scalar sum of the transverse momenta $\sum P_{T}$ for the multi-lepton events.

in mutually exclusive classes, depending on the type and number of high- $P_{T}$ objects found. In general a good agreement with the SM is found in the number of events for the selected topologies, Fig 2. Deviations from the SM are also searched for in the $\sum P_{T}$ and invariant mass $M_{\text {all }}$ distributions. All the observed deviations are compatible with the expected statistical fluctuations.

\section{CONCLUSION}

The H1 and ZEUS collaboration have analyzed the data collected between 1992 and 2007 at the HERA $e^{ \pm} p$ collider, in the search for physics beyond the Standard Model. Many different final states have been studied. In general a good agreement between data and the Standard Model simulations is observed. In some few cases deviations are observed, but still compatible with statistical fluctuations.

\section{REFERENCES}

1. I. Abt, et al., Nucl. Instrum. Meth. A386, 310-347 (1997).

2. I. Abt, et al., Nucl. Instrum. Meth. A386, 348-396 (1997).

3. The ZEUS detector, Status Report (unpublished), DESY (1993).

4. ZEUS Collaboration, Search for contact interactions, leptoquarks, large extra dimensions, and finite quark radius in ep collisions (2007), ZEUS-prel-07-028.

5. H1 Collaboration, High $Q^{2} \mathrm{NC}$ analysis using the complete HERA data (2007), H1prelim-07-141.

6. C. Adloff, et al., Phys. Lett. B568, 35-47 (2003), hep-ex/ 0305015.

7. H1 Collaboration (2009), 0904.3392.

8. F. D. Aaron, et al., Phys. Lett. B666, 131-139 (2008), 0805.4530.

9. F. D. Aaron, et al., Phys. Lett. B663, 382-389 (2008), 0802.1858.

10. W. Buchmuller, R. Ruckl, and D. Wyler, Phys. Lett. B191, 442-448 (1987).

11. H1 Collaboration, A search for leptoquarks in ep collisions at HERA (2007), H1 prelim-07-164.

12. F. D. Aaron, et al. (2009), 0901.0488. 

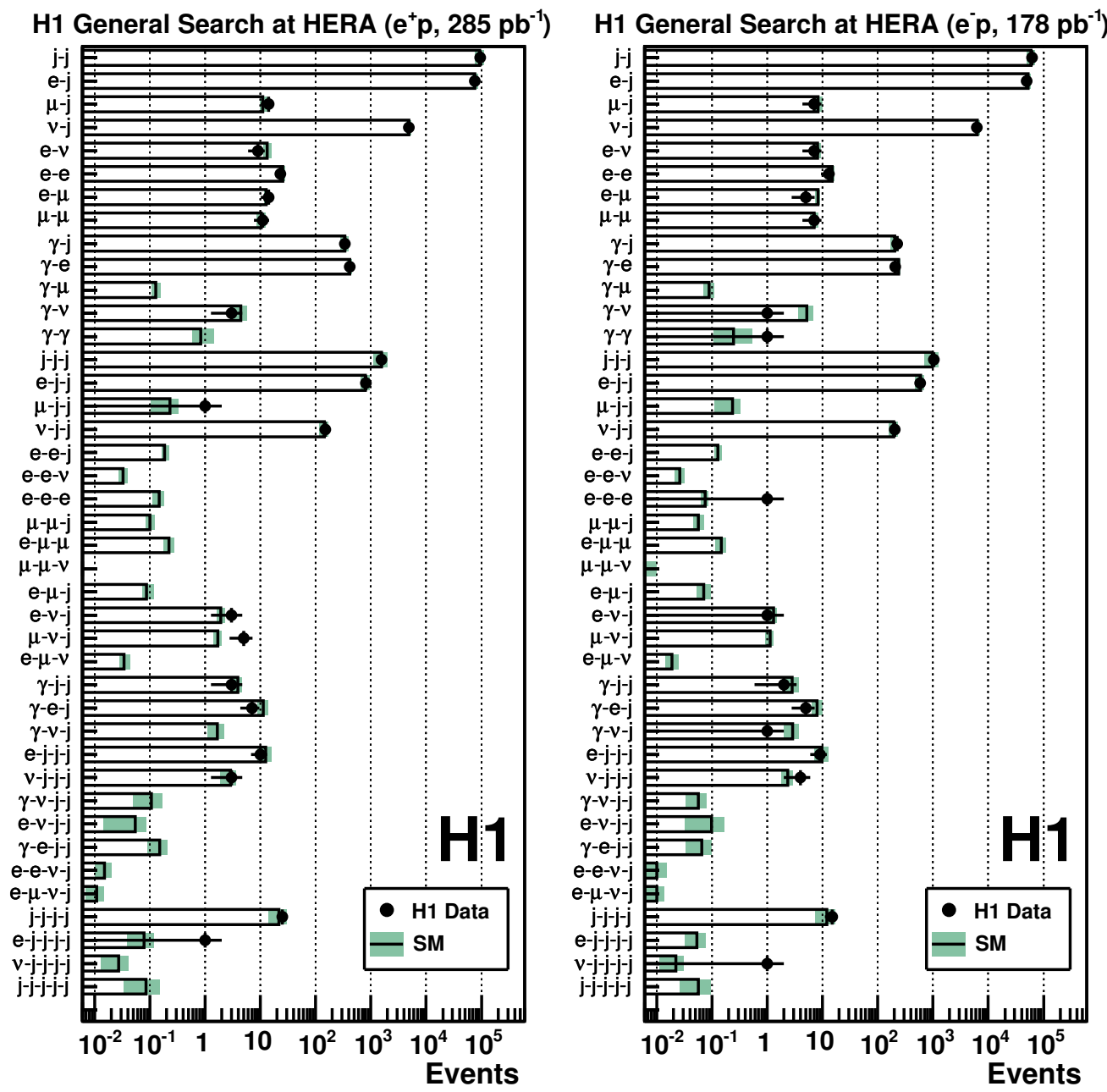

FIGURE 2. The data and SM expectation for all event classes with high- $P_{T}$ objects, where at least 0.01 events are observed or expected.

13. S. Chekanov, et al., Phys. Lett. B672, 106-115 (2009), 0807 . 0589.

14. H1 Collaboration (2009), 0904.3876.

15. ZEUS Collaboration, Search for single-top production in ep collisions at HERA (2009), ZEUS-prel09-009.

16. H1 and ZEUS Collaborations, Multi-leptons with high transverse momentum at HERA (2009), H1 prelim-09-064, ZEUS-prel-09-008.

17. F. D. Aaron, et al., Phys. Lett. B674, 257-268 (2009), 0901.0507. 